\title{
Comparison of maternal characteristics, pregnancy course, and neonatal outcome in preterm births with and without prelabor rupture of membranes
}

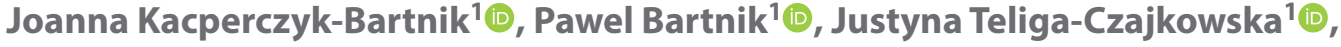 \\ Aneta Malinowska-Polubiec' ${ }^{10}$, Agnieszka Dobrowolska-Redo' ${ }^{\mathbb{D}}$,

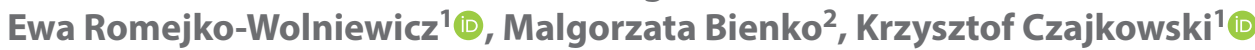 \\ ${ }^{1} 2^{\text {nd }}$ Department of Obstetrics and Gynecology, Medical University of Warsaw, Warsaw, Poland \\ ${ }^{2}$ Students'Scientific Group affiliated to $2^{\text {nd }}$ Department of Obstetrics and Gynecology, \\ Medical University of Warsaw, Warsaw, Poland
}

\begin{abstract}
Objectives: The aim of this study was to evaluate pregnancy outcome of patients with prelabor rupture of membranes receiving expectant management and giving birth prematurely in comparison to preterm births of patients with intact membranes.

Material and methods: It was a retrospective cohort study comparing maternal and neonatal outcome in two groups of preterm births. The first group included 299 consecutive singleton preterm births complicated by prelabor rupture of membranes. The second group consisted of 349 consecutive singleton preterm births without prelabor rupture of membranes. Results: Patients without PPROM underwent Caesarean sections more often than women from the pPROM group (65.3\% vs $45.2 \% ; p<0.001)$. No statistically significant differences regarding the gestational age during delivery were identified. Lower birth weight was detected in the group with no history of PPROM $(p<0.001)$.

No differences regarding early-onset sepsis were identified and higher percentage of late-onset infections was observed in infants with no history of pPROM $(8.9 \%$ vs $4.7 \% ; p=0.04)$. Pulmonary hypertension was more common in the infants from the PPROM group ( $4 \%$ vs $1.4 \% ; p=0.049$ ). Neonatal respiratory distress syndrome and respiratory failure were more prevalent in cases of no pPROM history $-20 \%$ vs $12.7 \%(p=0.02)$ and $40 \%$ vs $25.8 \%(p<0.001)$, respectively.

Conclusions: Development of multiple complications in preterm neonates may be more associated with the management, gestational age at birth, and birth weight than with the occurrence of preterm prelabor rupture of membranes.

Key words: pregnancy complications; pregnancy outcome; premature birth; preterm premature rupture of the membranes

Ginekologia Polska 2020; 91, 9: 528-538
\end{abstract}

\section{INTRODUCTION}

Prematurity remains the leading cause of neonatal morbidity and mortality worldwide [1]. Preterm prelabor rupture of membranes (pPROM) complicates about $3 \%$ of all pregnancies and one-third of pregnancies delivered before term [2]. Even though this complication has been managed for decades, available data on the results of planned early birth compared to expectant management of PPROM do not strongly support any method [3, 4].

\section{Objective}

The aim of this study was to evaluate maternal and neonatal outcome of preterm births complicated by PPROM and receiving expectant management in comparison to preterm births with intact membranes.

\section{MATERIAL AND METHODS}

Patient population and data collection

It was a retrospective cohort study comparing maternal and neonatal outcome in two groups of preterm births. The first group included 299 consecutive singleton preterm births complicated by preterm prelabor rupture of membranes. The second group consisted of 349 consecutive singleton preterm births without preterm prelabor rupture of membranes. Patients with fetal congenital defects and multiple pregnancies were excluded from the study. Data was collected from 
maternal and neonatal records of patients managed in a tertiary referral obstetric center between October 2016 and December 2018. Maternal obstetric history, comorbidities, pregnancy course, cervical microbiome, applied management, and delivery mode were examined. Neonatal outcome analysis included neonate's condition, birth weight, length of hospitalization, management in the Neonatal Intensive Care Unit (NICU), and type of applied treatment.

\section{Patients' management}

Preterm prelabor rupture of membranes was diagnosed with a rapid test detecting insulin-like growth factor binding protein (IGFBP-1) and with ultrasound examination. In case of positive result, expectant management was introduced. Cervical specimen collected on admission was cultured and prophylactic antimicrobial agents were administered. The antimicrobial regimen was established based on the local epidemiological data and consisted of intravenous cefuroxime for 10 days. The medication was adjusted in case of drug allergy or detected antimicrobial resistance. Cervical swabs were repeated every two weeks and further treatment was determined depending on the culture results. If no pathological agents were identified, the antimicrobial treatment was withdrawn after administering the prophylactic dose. Serum inflammatory markers were monitored every 2 days. Amniotic fluid index was measured twice a week.

Both groups - with and without PPROM — were monitored with ultrasound examinations and nonstress tests. In case of contractions before completed $35^{\text {th }}$ gestational week, accompanied by normal levels of inflammatory markers and normal nonstress test results, intravenous tocolysis was introduced. Antenatal corticosteroids were administered for better fetal maturation in case of preterm birth.

Premature infants were screened for early onset infections by monitoring of inflammatory markers, blood culture, and general condition. Prophylactic antimicrobial management included ampicillin and gentamicin for 48-72 hours.

\section{Statistical analysis}

Statistical analysis was performed using Statistica 13 (StatSoft. Inc.). T-Student test was used for quantitative data comparison between two groups. Two-sided Fisher's exact test was used for comparison of discrete variables. Logistic regression models were employed for multivariable analysis. $P$ value $<0.05$ was considered significant.

\section{RESULTS \\ Maternal characteristics}

Results of compared maternal characteristics are presented in Table 1. No differences between patients with PPROM and with intact membranes regarding maternal age, parity, and gestational age were observed. One of the

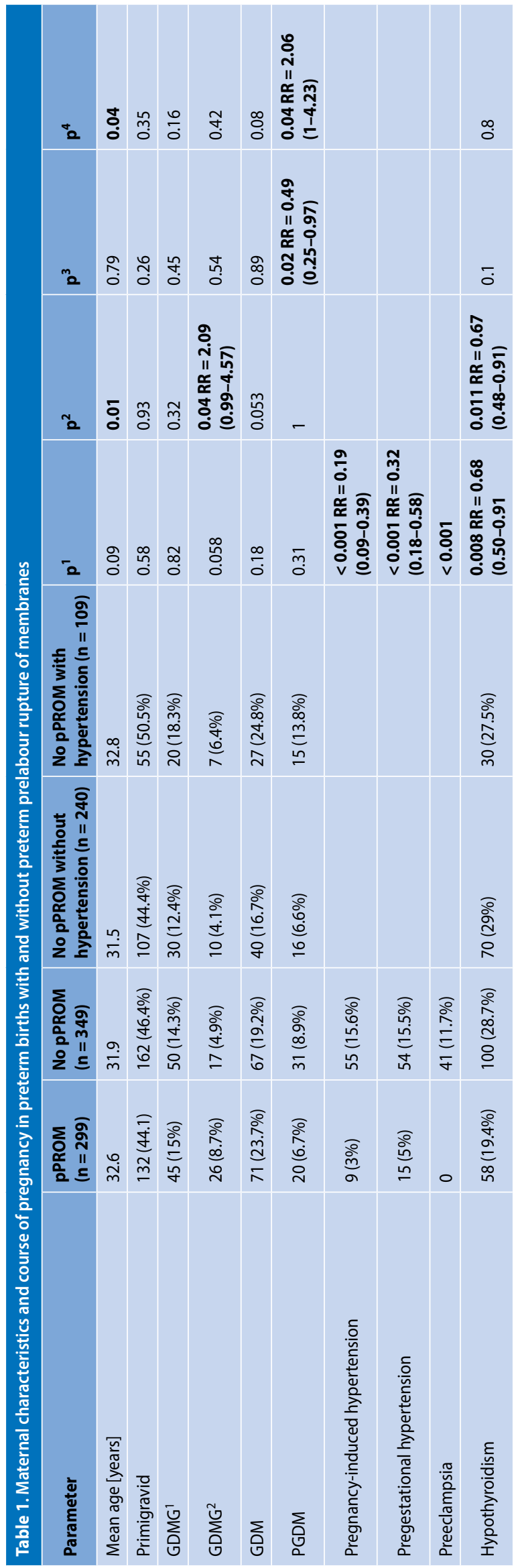




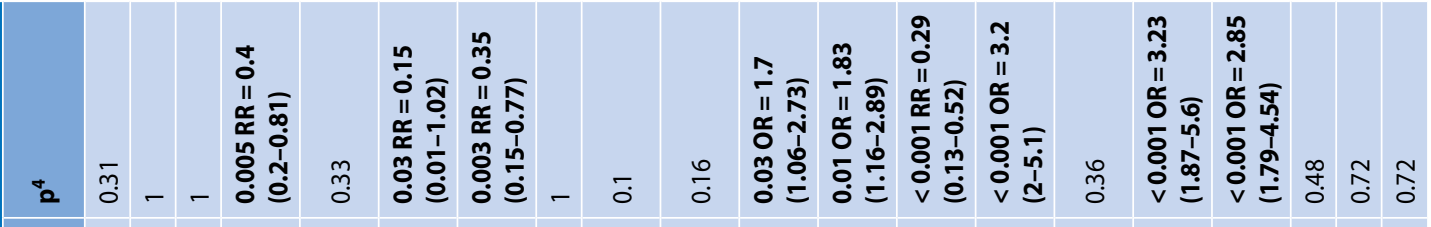

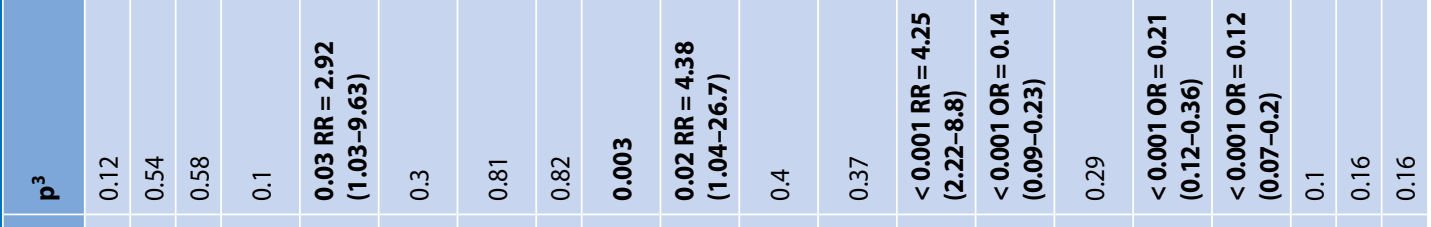

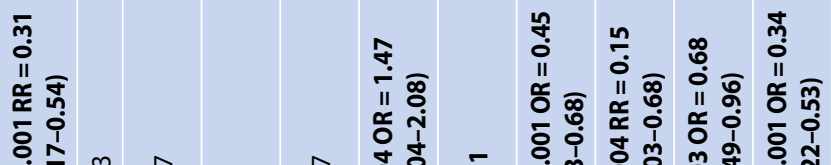

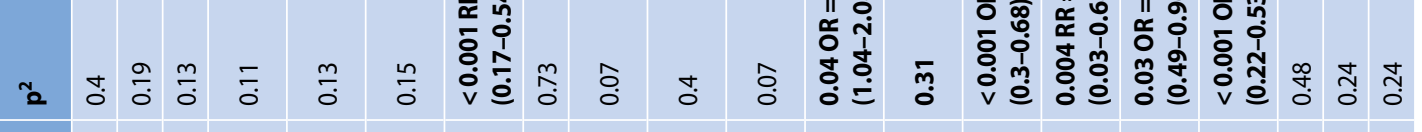




\begin{tabular}{|l|l|l|l|}
\hline \multicolumn{4}{|l}{$\begin{array}{l}\text { Table 2. Vaginal microbiome detected in patients from the pPROM } \\
\text { and no pPROM group }\end{array}$} \\
\hline Pathogen & With pPROM n (\%) & Without pPROM n (\%) & p \\
\hline Positive culture & $130(43.5)$ & $157(45)$ & 0.8 \\
\hline E. coli & $43(14.4)$ & $42(12)$ & 0.4 \\
\hline S. agalactiae & $39(13)$ & $58(16.6)$ & 0.2 \\
\hline C. albicans & $31(10.4)$ & $48(13.8)$ & 0.2 \\
\hline P. bivia & $19(6.4)$ & $19(5.4)$ & 0.7 \\
\hline E. faecalis & $17(5.7)$ & $14(4)$ & 0.4 \\
\hline G. vaginalis & $7(2.3)$ & $9(2.6)$ & 1 \\
\hline K. pneumoanie & $5(1.7)$ & $5(1.4)$ & 1 \\
\hline E. cloacae & $3(1)$ & $3(0.9)$ & 1 \\
\hline Ureaplasma & $3(1)$ & $6(1.7)$ & 0.5 \\
\hline P. melanogenica & $2(0.7)$ & 0 & 0.2 \\
\hline S. pyogenes & $2(0.7)$ & 0 & 0.2 \\
\hline B. fragilis & $1(0.3)$ & 0 & 0.5 \\
\hline B. ovatus & $1(0.3)$ & 0 & 0.5 \\
\hline C. crusei & $1(0.3)$ & 0 & 0.5 \\
\hline Cglabrata & $1(0.3)$ & $4(1.1)$ & 0.4 \\
\hline C. lusitaniae & $1(0.3)$ & $1(0.3)$ & 1 \\
\hline C. freundii & $1(0.3)$ & 0 & 0.5 \\
\hline C. krosei & $1(0.3)$ & $1(0.3)$ & 1 \\
\hline H. influenzae & $1(0.3)$ & 0 & 0.5 \\
\hline P. mirabilis & $1(0.3)$ & 0 & 0.5 \\
\hline & & & \\
\hline
\end{tabular}

most relevant differences regarded the occurrence of hypertensive disorders - 24 (8\%) patients in the PPROM group and $109(31 \%)$ in the second group $(p<0.001)$. Therefore, additional subgroups in the no pPROM group were analyzed depending on the hypertensive status in order to avoid bias resulting from different hypertensive disorders incidence between PPROM and no PPROM group [5].

Patients with $\mathrm{PPROM}$ more frequently had undergone cervical conization in the past ( $1.3 \%$ vs $0 ; p=0.045$ ). Obstetric pessary had been also more often used during earlier stages of pregnancies complicated by pPROM ( $10.7 \%$ vs $5.7 \%$; $p=0.03)$. Occurrence of cervical insufficiency and treatment with cervical cerclage was similar in both PPROM and no PPROM groups. Gestational diabetes treated with insulin occurred more frequently in the pPROM group compared to no PPROM, no hypertension group $(p=0.04)$. Hypothyroidism $(28.7 \%$ vs $19.4 \% ; p=0.008), 3^{\text {rd }}$ trimester vaginal bleeding $(14.6 \%$ vs $5.7 \% ; p<0.001)$, and stillbirth ( $3.7 \%$ vs $0.7 \% ; p=0.02$ ) were more frequent in the preterm pregnancies without PPROM. No differences between $\mathrm{PPROM}$ and no PPROM groups regarding the incidence of gestational diabetes treated with diet, asthma, and intrahepatic cholestasis of pregnancy were identified.

Similarly, no statistically relevant differences in the cervical microbiome among two groups were detected. Both the occurrence of positive culture result and the microbiome composition were comparable. Detected pathogens included E. coli, S. agalactiae, C. albicans, P. bivia, E. faecalis, G. vaginalis, K. pneumoanie, E. cloacae, Ureaplasma, P. melanogenica, S. pyogenes, B. fragilis, B. ovatus, C. crusei, C. glabrata, C. Iusitanie, C. freundii, C. krosei, H. influenzae, and P. mirabilis. Detailed information on the microbiome is presented in Table 2 .

\section{Management during pregnancy}

Distribution of antenatal corticosteroids administration was similar in both groups. However, a difference was identified concerning administration of full prophylactic dose followed by birth within ten days. Patients with pPROM more often received full prophylaxis (46.8\%) than patients with preterm birth uncomplicated by PPROM nor hypertension (37.3\%) ( $p=0.04)$. Intravenous tocolysis (fenoterol, atosiban) was more frequently required and administered in the pPROM patients ( $35.1 \%$ vs $23.8 \%$; $p=0.002$ ). Monitoring with the nonstress test resulted in detection of significantly more abnormalities in patients without PPROM than with the amniotic fluid leakage ( $38.7 \%$ vs $16 \% ; p<0.001)$.

\section{Mode of delivery}

No statistically significant differences regarding the gestational age during delivery were identified.

Patients without pPROM more often underwent Caesarean sections than women from the PPROM group $(65.3 \%$ vs $45.2 \% ; p<0.001)$. The difference between emergency Cesarean section was even higher. Almost $40 \%$ of patients from the no pPROM group underwent emergency Cesarean section - $31.5 \%$ in the hypertension negative subgroup and $56.8 \%$ in the hypertension positive subgroup. The high Cesarean section rate resulted from high number of medically indicated preterm deliveries in this group.

Every patient undergoing a Cesarean section had her amniotic fluid collected and cultured. Results showed more frequent prevalence of positive amniotic fluid culture in the pPROM group (6.4\%) than in the second group $(2 \%)(p=0.008)$.

\section{Birth weight}

There was a difference regarding mean birth weight, prevalence of small for gestational age under $10^{\text {th }}$ percentile $(2.7 \%$ in pPROM group vs $8.9 \%$ in no pPROM group; $p=0.001$ ); extremely low birth weight between 750 and $1000 \mathrm{~g}$ (4.3\% in pPROM group vs $8.6 \%$ in no pPROM group; $\mathrm{p}=0.04)$, and incredibly low birth weight under $750 \mathrm{~g}(2.7 \%$ in PPROM group vs $8.3 \%$ in no $\mathrm{PPROM}$ group; $\mathrm{p}=0.002$ ). Lower birth weight was detected in the group with no history of pPROM ( $p<0.001)$. The Ponderal index was not significantly different, however lower values were observed in the PPROM group and the no pPROM hypertension positive subgroup (Tab. 3). 


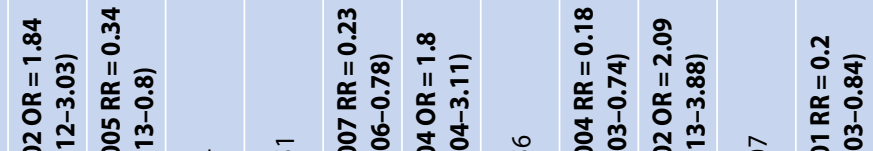

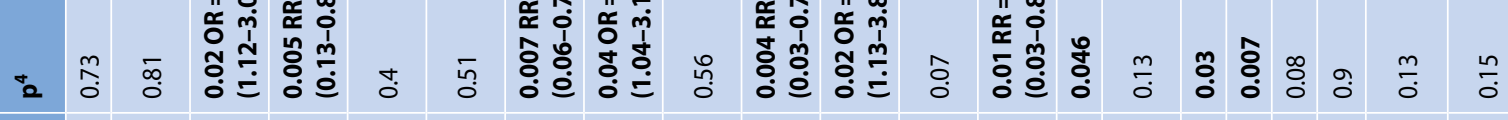
茟

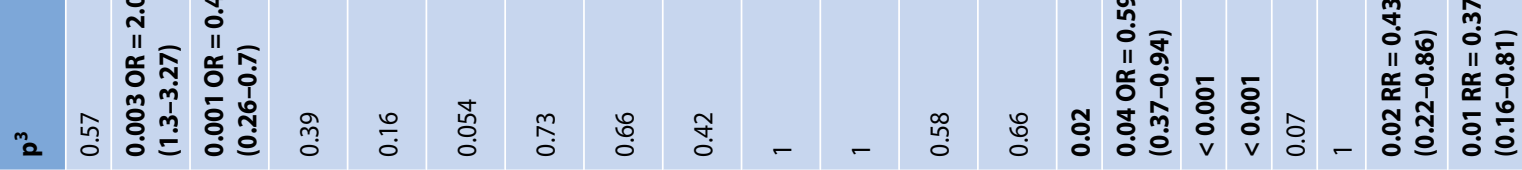

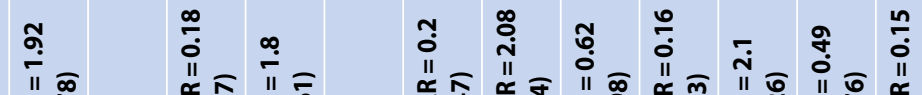

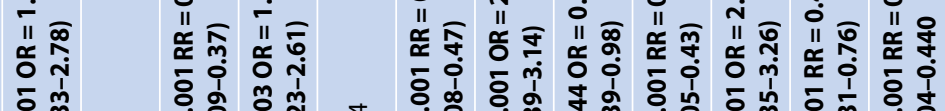

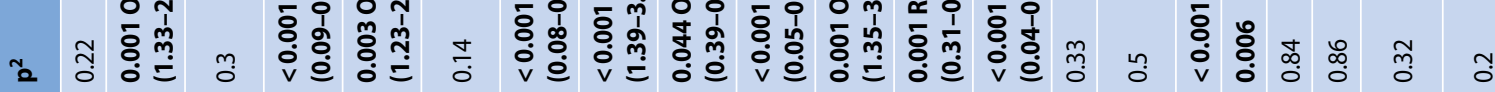

萡

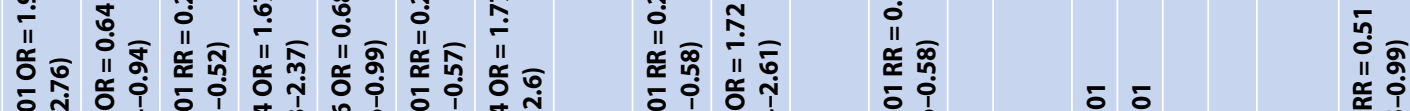

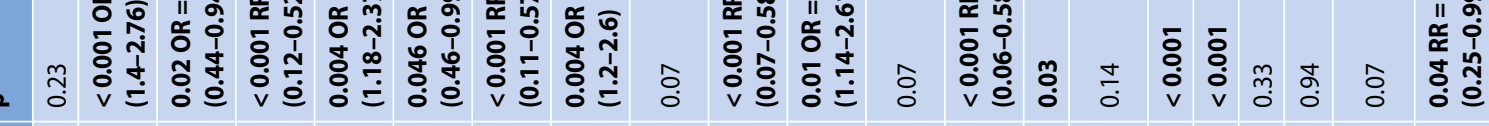

อิ

童

$\sum^{\frac{1}{3}} \frac{\sigma_{0}}{4}$

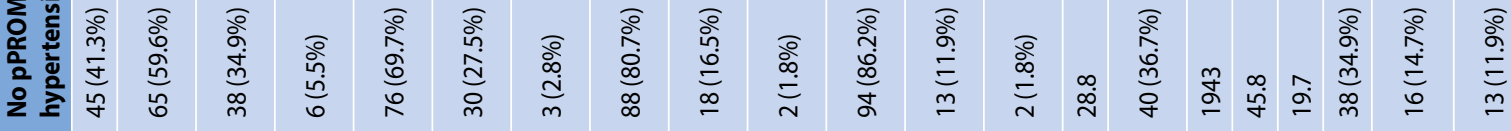

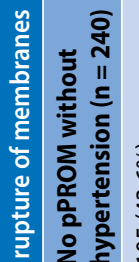

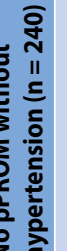

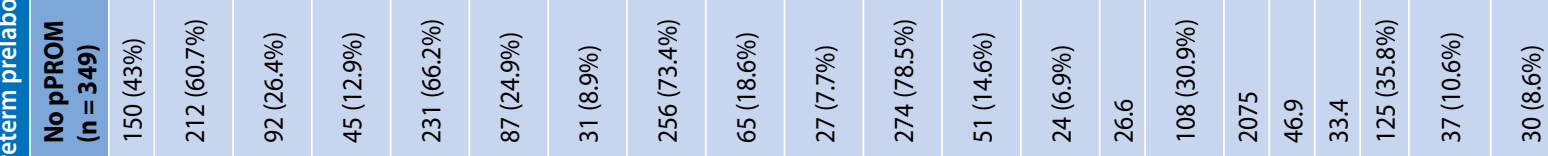

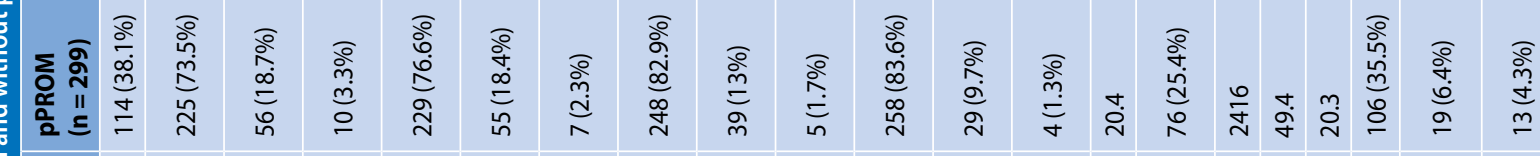

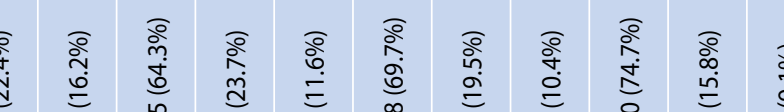

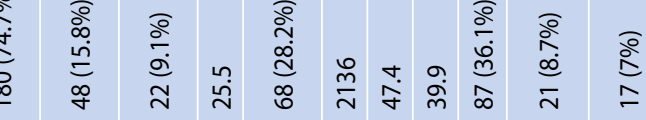




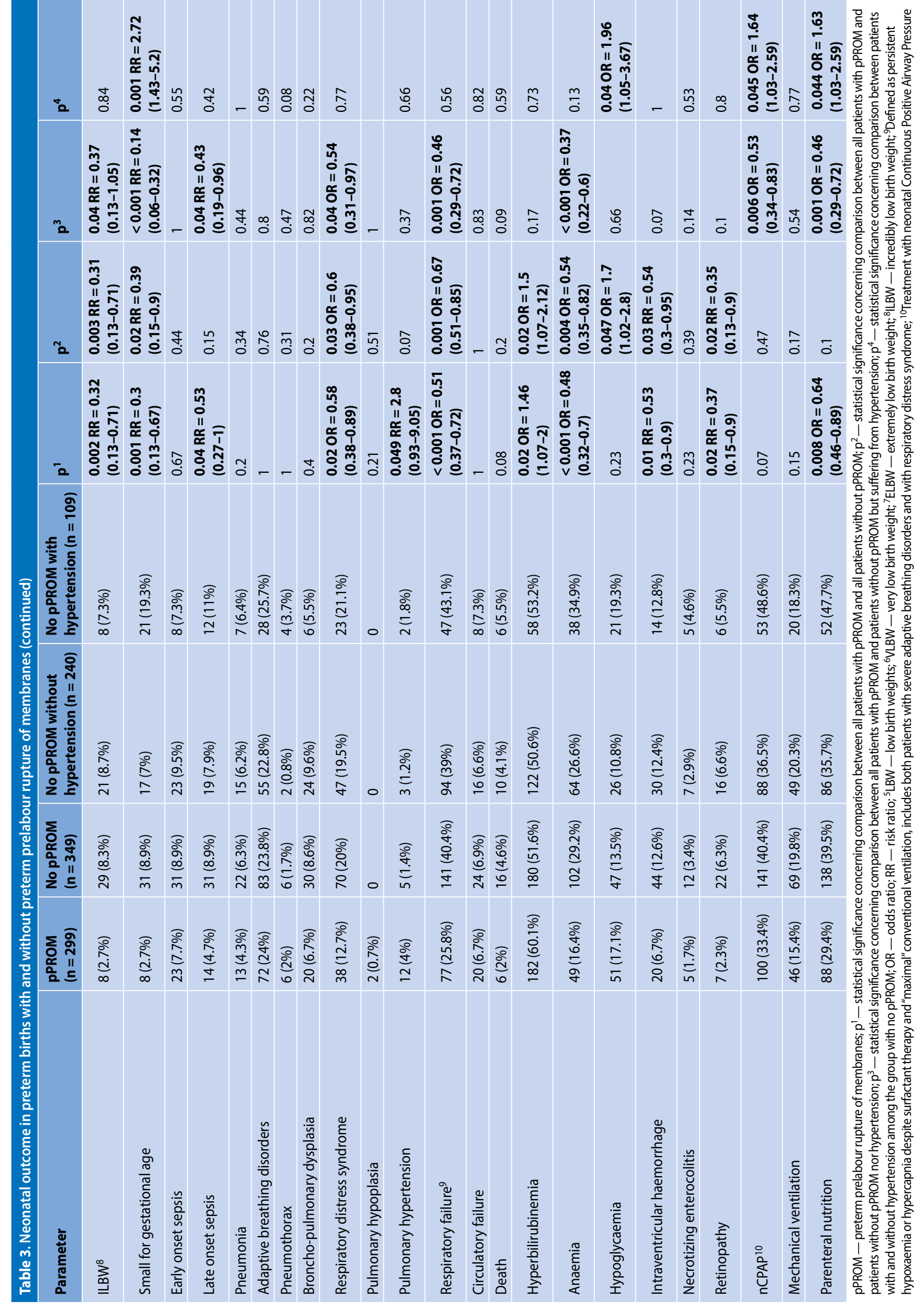




\section{Infections}

On the basis of the nonstress test (fetal tachycardia), elevated maternal body temperature, maternal heart rate and inflammatory markers, patients with PPROM were more often suspected of developing intrauterine infections (8\%) compared to the no pPROM group (4.6\%), however this difference was not statistically significant $(p=0.07)$. This observation did not correspond with the results of early or late-onset sepsis rates in the neonates. No differences regarding early-onset sepsis were identified and higher percentage of late-onset infections was observed in infants with no history of pPROM ( $8.9 \%$ vs $4.7 \% ; p=0.04$ ) (Tab. 3 ). The largest difference was observed between infants of patients with PPROM and patients without PPROM but with hypertension (4.7\% vs 11\%). Figures 1 and 2 show the distribution of early and late-onset sepsis depending on the gestational week at birth and pPROM status. No difference in gestational age at birth among mentioned subgroups were identified.

\section{Neonatal respiratory complications}

Pulmonary hypertension was more common in the infants from the pPROM group ( $4 \%$ vs $1.4 \% ; p=0.049$ ). Surprisingly, the distribution of other respiratory complications was similar between the groups or higher in the group with no history of pPROM (Tab. 3). Identified statistically significant differences regarded the occurrence of respiratory failure, which was defined as persistent hypoxemia or hypercap-

\section{EOS and LOS in PROM group}

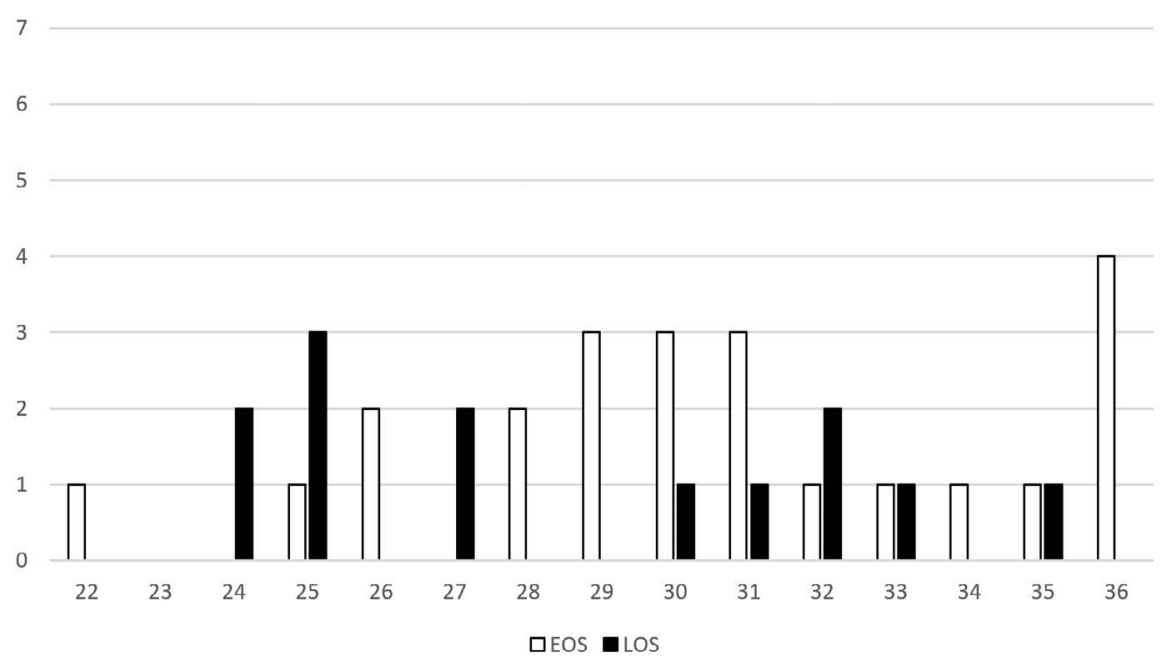

Figure 1. Occurrence of early onset sepsis and late-onset sepsis in the pPROM group depending on the gestational week at birth

EOS and LOS in no PROM group

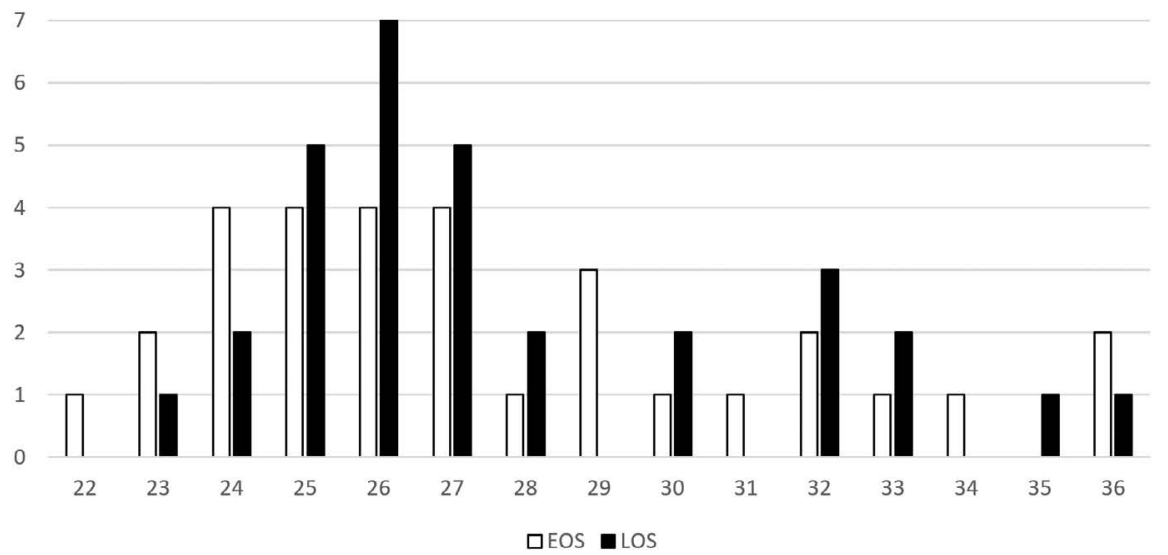

Figure 2. Occurrence of early onset sepsis and late-onset sepsis in the no pPROM group depending on the gestational week at birth 
nia despite surfactant therapy and "maximal" conventional ventilation and included both patients with severe adaptive breathing disorders and with respiratory distress syndrome. This condition affected over $40 \%$ of infants in the no PPROM group and $25.8 \%$ of infants from the PPROM group $(p<0.001)$. Neonatal respiratory distress syndrome was also more prevalent in case of no pPROM history ( $20 \%$ vs $12.7 \%$; $p=0.02$ ). The prevalence of neonatal respiratory distress syndrome in both PPROM and no pPROM groups depending on the gestational age at birth is presented in Figure 3 and Figure 4. No statistically significant differences in occurrence of pulmonary hypoplasia, bronchopulmonary dysplasia, use of neonatal continuous positive airway pressure (nCPAP) nor mechanical ventilation between the PPROM and no PPROM group during the observation time were detected.

\section{Other neonatal complications}

Preterm neonates of patients without pPROM obtained more frequently lower Apgar score results, more often developed intraventricular hemorrhage $(p=0.01)$, retinopathy $(p=0.02)$, and anemia $(p<0.001)$, required longer infant's hospitalization $(p=0.03)$, and parenteral nutrition $(p=0.008)$ (Tab. 3). Hyperbilirubinemia was more frequently diagnosed in the pPROM group $(p=0.02)$. Figures 5 and 6 show the distribution of neonatal complications depending on the gestational age at birth.

\section{Multivariable analysis}

In the bi-variable logistic regression models PPROM was not associated with the occurrence of early-onset sepsis or the late-onset sepsis after adjustment for ges-

\section{Neonatal RDS in PROM group}

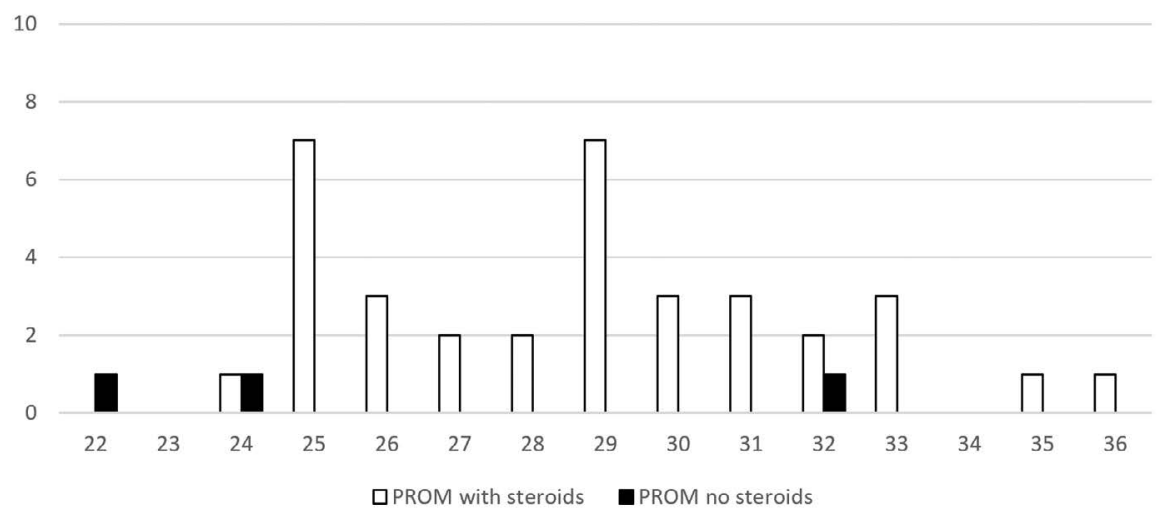

Figure 3. Neonatal Respiratory Distress Syndrome in the pPROM group depending on the gestational week at birth

\section{Neonatal RDS in no PROM group}

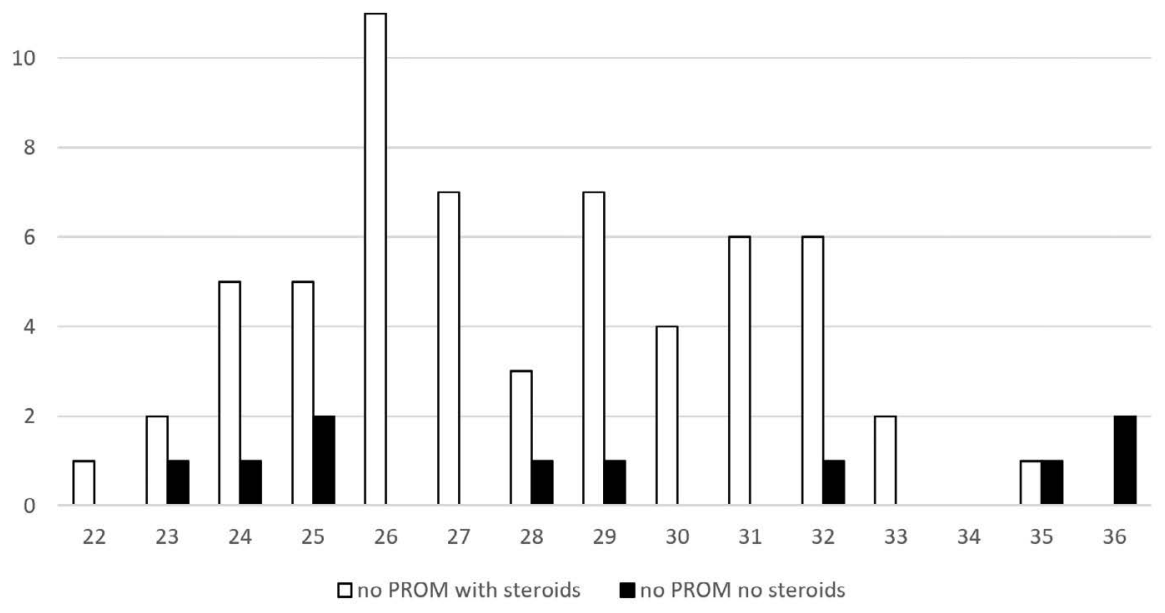

Figure 4. Neonatal Respiratory Distress Syndrome in the no pPROM group depending on the gestational week at birth 


\section{Complications in pPROM group}

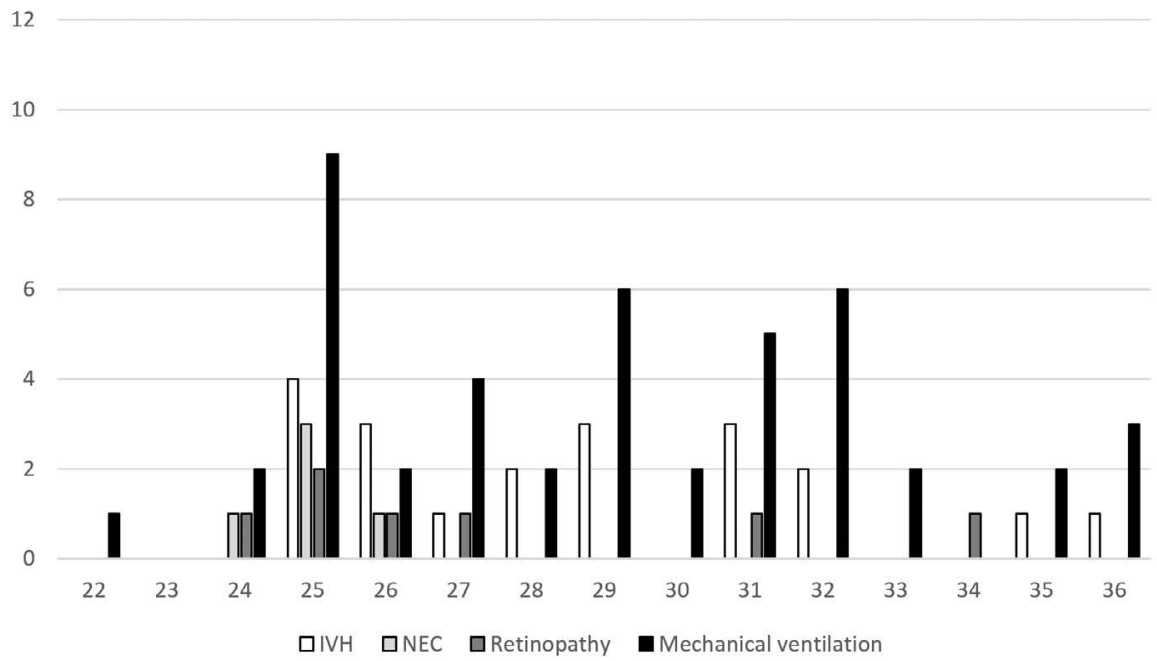

Figure 5. Occurrence of neonatal complications in the PPROM group depending on the gestational week at birth

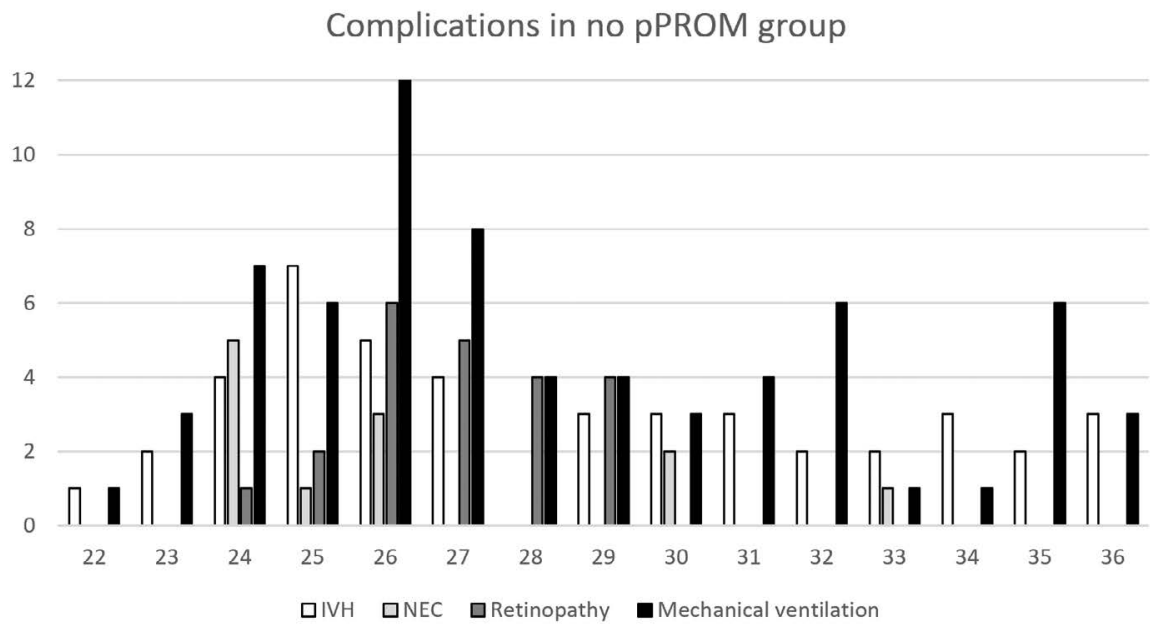

Figure 6. Occurrence of neonatal complications in the no pPROM group depending on the gestational week at birth

tational age at birth $(p<0.001)$ or lower birth weight $(p<0.001)$.

Similarly, the occurrence of the respiratory distress syndrome was independent from pPROM after adjustment for gestational age at birth $(p<0.001)$ or lower birth weight $(p<0.001)$.

PPROM occurrence was associated with reduced probability of respiratory failure (odds ratio 0.44 with $95 \%$ confidence interval 0.29-0.66; $p<0.001$ ) even after adjustment for gestational age at birth $(p<0.001)$.

\section{DISCUSSION}

Certain differences regarding maternal comorbidities and past medical history, pregnancy course, delivery mode, and neonatal complications between patients giving birth before term with pPROM and with intact membranes, were identified in this study.

The largest difference in maternal comorbidities was observed in case of the prevalence of pregnancy-induced hypertension ( $3 \%$ vs $15.6 \%$ ), pregestational hypertension ( $5 \%$ vs $15.5 \%$ ), and preeclampsia (0 vs $11.7 \%$ ), which were less common in the PPROM group. Analogous results were reported by other authors: $5.8 \%$ vs $7.6 \%$ of hypertensive disorders in the Bouvier et al. study [6] and $14.5 \%$ vs $52 \%$ of pregnancy-induced hypertension incidence in the PPROM and control groups described by Dannapaneni et al. [7]. Similarly, Pharande et al. observed lower prevalence of hypertensive disease of pregnancy in patients with PPROM 
than with the intact membranes (7.6\% vs $26.7 \%)$ [8]. Hypothyroidism was also more prevalent in the group without pPROM (19.4\% vs $28.7 \%$ ), which stands in contrast to results of other studies. In a systematic review by Maraka et al., the pooled relative risk of prelabor rupture of membranes in women with subclinical hypothyroidism was $1.43 \%$ according to data from six randomized trials and cohort studies [9]. In a prospective cohort study by Johnson et al. patients with subclinical hypothyroidism presented significantly more frequent history of PPROM (13\%) compared to euthyroid women (1.4\%) [10]. However, most studies indicate association between hypothyroidism and increased risk of prematurity, which might explain different proportions in our study focusing exclusively on the analysis of patients with preterm births [11]. Bouvier et al. emphasized the meaning of gestational diabetes in possible pathogenesis of PPROM due to sterile inflammation as they reported both higher rates of gestational diabetes and insulin intake in the PPROM group [6]. Our results confirm these findings only partially as insulin intake was more common among patients with PPROM (8.7\%) than among the subgroup without PPROM and without hypertension (4.1\%). No other significant differences in gestational diabetes incidence were observed.

Analyzed past medical history included treatment for cervical intraepithelial lesion (CIN) before pregnancy. In a systematic review based on 21 observational studies, the relative risk of pPROM was enhanced in these women (RR 2.36) [12]. Our results confirm this statement as the incidence of conization before gestation was more frequent in the PPROM group. In a recent study by Maina et al. the incidence of pPROM among patients with the history of excisional treatment was also increased (13.13\% vs 2.71\%) [13]. Another analyzed factor was cervical insufficiency in current pregnancy. Differences in cervical insufficiency and treatment with cervical cerclage were not significant, but the positive history of pessary application in current pregnancy was higher in the PPROM group.

Gestational age at birth did not differ between the groups. However, the group without pPROM was characterized by lower birth weight, higher occurrence of small for gestational age, extremely low birth weight, and incredibly low birth weight. These differences were still significant after subgroup analysis with the consideration of hypertensive disorders distribution. This was not observed in the Pharande et al. study, but comparable results were obtained by other authors $[8,14]$.

Pathological cervical microbiome was detected in $43.5 \%$ patients with pPROM and $45 \%$ without pPROM. In a study by Swiatkowska-Freund et al. the incidence of positive cervical swabs in the pPROM group was 49\% [15]. As the prevalence concerns almost half of the patients, these results support introduction of standard antimicrobial prophylaxis as prevention of ascending infection route due to ruptured membranes. In this study mentioned prophylaxis was introduced in every patient with PPROM on admission or after pPROM occurrence during earlier hospitalization. Combined with strict monitoring of maternal inflammation parameters and fetal well-being, this early antimicrobial intervention, adjusted when needed after the antibiogram results analysis, could be the reason that the early onset-sepsis (EOS) rates were similarly distributed among the groups with and without PPROM (7.7\% vs $8.9 \%)$. Similar observations regarding prophylaxis utility can be made based on other studies, in which antimicrobial prophylaxis was introduced in less than $50 \%$ or in over $80 \%$ of patients and the rates of EOS cases strongly varied $[8,15]$. What is more, in a study by Hanke et al., it was proved in a multivariable logistic regression that $\mathrm{PPROM}$ is not an independent risk factor for EOS in infants with extremely low birth weight [16]. The proportion of infants exposed to prenatal antibiotic treatment in this study exceeded $80 \%$. The number of neonates exposed to tocolytic treatment of mothers was also relatively high (68.1\%) and higher than in the control group. Concerning the low EOS rate and more frequent use of intravenous tocolysis in patients with PPROM than with the intact membranes, our results are consistent with other studies reporting administration of intravenous tocolysis in pPROM $[16,17]$.

The occurrence of late-onset sepsis (LOS) in our study was higher in the group of premature infants without the history of pPROM. The association between LOS incidence, pPROM occurence, gestational age at birth and birth weight were examined in a multivariable analysis in order to determine LOS risk factors. Eventually, no relation between LOS incidence and PPROM was observed. This indicates more relevant factors associated with LOS other than PPROM. As the mean hospitalization duration of infants from the no pPROM group was significantly longer, it is possible that the risk of LOS depends on the number of required neonatal procedures and time of observation under strict medical supervision.

Regarding the incidence of neonatal respiratory complications, our study showed increased risk of pulmonary hypertension in the PPROM group. As patients with fetal congenital defects were excluded from the study, this result does not represent pulmonary hypertension caused by diagnosed congenital heart disease. This result is also compatible with other studies reporting compromised lung development due to oligohydramnios $[8,18,19]$. Pulmonary hypertension is also associated with prematurity and its treatment side effects e.g. by mechanisms including ventilator-induced lung injury and oxidant stress [20]. On the contrary, the incidence of respiratory distress syndrome (RDS) and respiratory failure was higher in the group with 
intact membranes $(12.7 \%$ vs $20 \%$ and $25.8 \%$ vs $40.4 \%$, respectively). This result could be associated with lower birth weight and increased cesarean section rate ( $45.2 \%$ vs $65.3 \%)$ including emergency cesarean sections ( $13.7 \%$ vs $39.5 \%)$ in the group without PPROM [21, 22]. Administration of antenatal corticosteroids, including the full prophylactic dose was comparable between the groups ( $46.8 \%$ vs $42 \%$ ). Multivariable analysis confirmed higher incidence of RDS associated with lower birth weight and earlier gestational age at birth.

\section{CONCLUSIONS}

Development of multiple complications in preterm neonates, including sepsis and respiratory complications, may be more associated with the management, gestational age, and birth weight than with the occurrence of preterm prelabor rupture of membranes.

\section{REFERENCES}

1. Lehtonen L, Gimeno A, Parra-Llorca A, et al. Early neonatal death: A challenge worldwide. Semin Fetal Neonatal Med. 2017; 22(3): 153-160, doi: 10.1016/j.siny.2017.02.006, indexed in Pubmed: 28238633.

2. Mercer BM. Preterm premature rupture of the membranes: current approaches to evaluation and management. Obstet Gynecol Clin North Am. 2005; 32(3): 411-428, doi: 10.1016/j.ogc.2005.03.003, indexed in Pubmed: 16125041 .

3. Bond DM, Middleton P, Levett KM, et al. Planned early birth versus expectant management for women with preterm prelabour rupture of membranes prior to 37 weeks' gestation for improving pregnancy outcome. Cochrane Database Syst Rev. 2017; 3: CD004735, doi: 10.1002/14651858.CD004735.pub4, indexed in Pubmed: 28257562.

4. Pasquier JC, Claris $\mathrm{O}$, Rabilloud $\mathrm{M}$, et al. Intentional early delivery versus expectant management for preterm premature rupture of membranes at 28-32 weeks' gestation: A multicentre randomized controlled trial (MICADO STUDY). Eur J Obstet Gynecol Reprod Biol. 2019; 233: 30-37, doi: 10.1016/j.ejogrb.2018.11.024, indexed in Pubmed: 30553135.

5. Shen M, Smith GN, Rodger M, et al. Comparison of risk factors and outcomes of gestational hypertension and pre-eclampsia. PLoS One. 2017; 12(4): e0175914, doi: 10.1371/journal.pone.0175914, indexed in Pubmed: 28437461.

6. Bouvier D, Forest JC, Blanchon L, et al. Risk Factors and Outcomes of Preterm Premature Rupture of Membranes in a Cohort of 6968 Pregnant Women Prospectively Recruited. J Clin Med. 2019; 8(11), doi: 10.3390/jcm8111987, indexed in Pubmed: 31731659.

7. Dannapaneni $\mathrm{N}$, Oleti T, Surapaneni $\mathrm{T}$, et al. Immediate neonatal outcomes of preterm infants born to mothers with preterm pre-labour rupture of membranes. Indian J Med Res. 2017; 146(4): 476-482.

8. Pharande $\mathrm{P}$, Mohamed AL, Bajuk B, et al. Preterm infant outcomes in relation to the gestational age of onset and duration of prelabour rupture of membranes: a retrospective cohort study. BMJ Paediatr
Open. 2017; 1(1): e000216, doi: 10.1136/bmjpo-2017-000216, indexed in Pubmed: 29637178.

9. Maraka S, Ospina NM, O'Keeffe DT, et al. Subclinical Hypothyroidism in Pregnancy: A Systematic Review and Meta-Analysis. Thyroid. 2016; 26(4): 580-590, doi: 10.1089/thy.2015.0418, indexed in Pubmed: 26837268.

10. Johnson N, Taylor-Christmas AK, Chatrani V, et al. Obstetric Outcomes of an Afro-Caribbean Cohort Following Universal Screening and Treatment of Subclinical Hypothyroidism. West Indian Med J. 2015; 65(1): 78-82, doi: 10.7727/wimj.2014.275, indexed in Pubmed: 26716797.

11. Lee SY, Cabral HJ, Aschengrau A, et al. Associations Between Maternal Thyroid Function in Pregnancy and Obstetric and Perinatal Outcomes. J Clin Endocrinol Metab. 2020; 105(5), doi: 10.1210/clinem/dgz275, indexed in Pubmed: 31838502.

12. Kyrgiou M, Athanasiou A, Kalliala IEJ, et al. Obstetric outcomes after conservative treatment for cervical intraepithelial lesions and early invasive disease. Cochrane Database Syst Rev. 2017; 11: CD012847, doi: 10.1002/14651858.CD012847, indexed in Pubmed: 29095502.

13. Maina $G$, Ribaldone $R$, Danese $S$, et al. Obstetric outcomes in patients who have undergone excisional treatment for high-grade cervical squamous intra-epithelial neoplasia. Eur J Obstet Gynecol Reprod Biol. 2019; 236: 210-213, doi: 10.1016/j.ejogrb.2019.02.025, indexed in Pubmed: 30922526.

14. Sae-Lin $P$, Wanitpongpan $P$. Incidence and risk factors of preterm premature rupture of membranes in singleton pregnancies at Siriraj Hospital. J Obstet Gynaecol Res. 2019; 45(3): 573-577, doi: 10.1111/jog.13886, indexed in Pubmed: 30537150.

15. Swiatkowska-Freund M, Traczyk-Łos A, Partyka A, et al. Perinatal outcome in preterm premature rupture of membranes before 37 weeks of gestation. Ginekol Pol. 2019; 90(11): 645-650, doi: 10.5603/GP.2019.0109, indexed in Pubmed: 31802465.

16. Hanke K, Hartz A, Manz M, et al. German Neonatal Network (GNN). Preterm prelabor rupture of membranes and outcome of very-low-birth-weight infants in the German Neonatal Network. PLoS One. $2015 ;$ 10(4): e0122564, doi: 10.1371/journal.pone.0122564, indexed in Pubmed: 25856083.

17. Chackowicz A, Czuzoj-Shulman N, Abenhaim HA. The effects of tocolysis on neonatal septic death in women with PPROM: a retrospective cohort study. Arch Gynecol Obstet. 2018; 298(5): 897-902, doi: 10.1007/s00404018-4871-9, indexed in Pubmed: 30206736.

18. Najrana T, Ramos LM, Abu Eid R, et al. Oligohydramnios compromises lung cells size and interferes with epithelial-endothelial development. Pediatr Pulmonol. 2017; 52(6): 746-756, doi: 10.1002/ppul.23662, indexed in Pubmed: 28152278.

19. Weiner E, Barrett J, Zaltz A, et al. Amniotic fluid volume at presentation with early preterm prelabor rupture of membranes and association with severe neonatal respiratory morbidity. Ultrasound Obstet Gynecol. 2019; 54(6): 767-773, doi: 10.1002/uog.20257, indexed in Pubmed: 30834608.

20. Steinhorn RH. Neonatal pulmonary hypertension. Pediatr Crit Care Med. 2010; 11(2 Suppl): S79-S84, doi: 10.1097/PCC.0b013e3181c76cdc, indexed in Pubmed: 20216169.

21. Condò V, Cipriani S, Colnaghi M, et al. Neonatal respiratory distress syndrome: are risk factors the same in preterm and term infants? J Matern Fetal Neonatal Med. 2017; 30(11): 1267-1272, doi: 10.1080/14767058.2016.1210597, indexed in Pubmed: 27399933.

22. Li Y, Zhang $C$, Zhang D. Cesarean section and the risk of neonatal respiratory distress syndrome: a meta-analysis. Arch Gynecol Obstet. 2019; 300(3): 503-517, doi: 10.1007/s00404-019-05208-7, indexed in Pubmed: 31187205 